\title{
A meta-analysis of ECG abnormalities (arrhythmia) in cardiomyopathies
}

\author{
Aref Albakri* \\ St-Marien hospital Bonn Venusberg, department of internal medicine, Bonn, Germany
}

\begin{abstract}
The ECG is an important initial test for patients suspected with CM. The greater majority of patients who meet the diagnostic criteria of the primary structural and functional forms of CM - DCM, HCM, RCM, LVNC, and ARVC usually present with ECG abnormalities. ECG tests are the mainstay for the detection of arrhythmias, which are frequent manifestations of cardiac involvement, and they portend poor prognosis and complicate clinical management. The most studied arrhythmias among $\mathrm{CM}$ patients are $\mathrm{AF}, \mathrm{VT}$ and extrasystoles. The overall prevalence of AF was $12.8 \%$. The highest prevalence was in RCM patients (19.2\%) followed by DCM (15.2\%), HCM (8.0\%), LVNC (7.7\%), and the least was in ARVC patients (3.3\%). The prevalence of VT was 38.0\%, which was highest in ARVC (55.1\%), followed by DCM (32.4\%), HCM (22.9\%), LVNC (20.2\%), RCM (10.1\%). Extrasystoles, specifically PVC had a prevalence of 56.6\%. DCM had the highest prevalence (79.2\%), ARVC (41.8\%), RCM (56.6\%), the one study investigating $177 \mathrm{HCM}$ patients reported 136 had PVCs. ECG-assessed VTS or PVCs is an important diagnostic tests to differentiated VTSs due to ARVD and those due to RVOT. In conclusion, ECG is a valuable test for AF, VT and PVC, which are prevalent and can inform the administration of the most appropriate therapy.
\end{abstract}

\section{Introduction}

The standard 12-lead electrocardiogram (ECG) is a useful tool for the initial cardiac assessment in patients suspected with a heart disease. It provides a bedside snapshot of the electrical activity of the heart including vector information such as $\mathrm{P}, \mathrm{QRS}$ and $\mathrm{T}$ waves. However, a snapshot of a few heart beats for some seconds is inadequate to provide a holistic assessment of the electrical activities of the heart because of the potential to overlook important conduction abnormalities [1]. The traditional Holter ECG that displays two or three leads can record cardiac activity continuously for 24 to 48 hours or longer depending on the type of monitoring used. However, its limited vector information is an important shortcoming in clinical diagnosis of heart diseases. The 12-lead Holter ECG overcomes these limitations and is preferred for the detection of arrhythmias and localizes the origin of arrhythmias [2]. Besides the detection of arrhythmia and electrical conduction abnormalities, the 12-lead Holter monitoring is useful for the evaluation of the therapeutic efficacy of drugs and other clinical interventions such as assessing the efficiency of bi-ventricular pacing in patients with heart failure (HF) and comorbid permanent atrial fibrillation (AF). In addition, the analysis of the parameters of 12-lead Holter monitoring can provide useful information for risk stratification and prognostication.

The ECG is a useful initial diagnostic tool for the assessment of cardiac function in patients suspected with cardiomyopathy (CM) - a spectrum, of heterogeneous myocardial disorders that can potentially impair the normal functioning of the heart. It is the most common cause of $\mathrm{HF}$ and a leading indication for cardiac transplantation as well as among the most common diagnoses requiring hospitalization $[3,4]$. Patients with CM are more likely to develop arrhythmias than in the general population, which confers a substantial risk of morbidity and mortality. The main cause of mortality in CM patients is end-organ dysfunction due to pump failure or arrhythmia-related deaths [4]. Despite considerable evidence that arrhythmias and sudden cardiac death are complicate the management of $\mathrm{CM}$, studies evaluating $\mathrm{CM}$ patients disproportionately focus on HF with little attention given to arrhythmias [5]. Studies on systemic evaluation of ECG in large cohorts of CM patients are lacking. Nonetheless, such studies may have important clinical implications since they may provide valuable diagnostic and prognostic information. The present study examines the prevalence of and types of arrhythmias in different morphofunctional forms of CM - dilated (DCM), hypertrophic (HCM), restrictive (RCM), and arrhythmogenic right ventricular CM (ARVC). Although various aetiological types of CM have been described, they lead to a common pathway of four morphological abnormalities (DCM, HCM, RCM, \& ARVC).

\section{Study selection}

The present meta-analysis aggregates current published evidence of the diagnostic value of ECG in patients with suspected or confirmed CM. More specifically, to address two questions. (1) What is the value of ECG in the diagnosis of arrhythmias? If so, what is the prevalence, and the most common types of arrhythmias affecting different structural forms of CM. (2) What are the implications of the findings to the diagnosis and treatment of patients with CM? The second question is based on findings that different structural forms of arrhythmias in patients with heart disease could have important diagnostic, prognostic and therapeutic implications. The search for relevant studies was performed on online database PubMed. In addition, key journals in the field of cardiology, top 100 results from Google Scholar, and references

${ }^{*}$ Correspondence to: Aref Albakri, St-Marien hospital Bonn Venusberg, department of internal medicine, Bonn, Germany, E-mail: arefalbakri@yahoo.com

Key words: cardiomyopathy, electrocardiography, arrhythmias, atrial fibrillation [af], ventricular tachycardia [vt], premature ventricular contractions [pvc]

Received: May 01, 2020; Accepted: May 15, 2020; Published: May 22, 2020 
of articles and review papers were handsearched. Studies were included if the diagnosis included ECG-detected arrhythmias. The patient population of interest were who have been diagnosed with one of the major structural forms of $\mathrm{CM}$. The outcome of interest were the types and prevalence of arrhythmias in different structural forms of CM.

\section{Study characteristics}

Forty (40) studies were included in the present meta-analysis and constituted the final dataset for a pooled analysis [5-44]. The studies were not region specific. They covered several different geographical regions including American, Europe and Asia. The specific countries included were China [43], Germany [6-8,29], India [9,11-13], Italy [5,15,27,28,31-33], Japan [41], Nepal [10], Netherlands [24,25], Norway [36], Spain [18], Sweden [42]. Switzerland [22], U.K. [14,16,17,23,44], U.S. [14,19-21,26,30,34,35,37-40]. The wide coverage of the studies is useful since they include patients exposed to different diagnostic approaches and environmental risk factors and the underlying aetiologies and thus, providing a more holistic picture.
The included studies also adopted difference research designs of which a greater number of studies $(n=29 ; 72.5 \%)$ were prospective cohort studies [5-9,13,15-25,28-34,36-38,42,44]. The remaining studies were retrospective cohort $(n=9 ; 22.5 \%)[10,14,26,27,35,39,40,41,43]$ and cross-sectional studies $(n=2 ; 5 \%)[11,12]$. In addition to study design, most of the studies were non-randomized and non-blinded because the primary aim of this study was to determine the prevalence and types of arrhythmias among CM patients. Moreover, the area or location of study also varied across the included studies, ranging from single-centre, multiple-centres and population-based studies.

Several types of CM exist based on structural and functional classification. However, structural forms of $\mathrm{CM}$ in which studies provide raw data on the presence and prevalence of arrhythmias were only five. These were DCM, HCM, RCM, ARVC, and LVNC. Of these five structural forms of CM, the most studied was ARVC evaluated by ten studies (25\%) [27-36], followed by DCM evaluated by nine studies (23\%) [5-13], RCM by eight studies (20\%) [37-44], HCM by seven studies (18\%) [14-20] and LVNC by six studies (15\%) (Table 1).

Table 1: Study characteristics

\begin{tabular}{|c|c|c|c|c|c|c|c|c|c|}
\hline 1st Author, Year & Country & Design & Cohort Type & Size & Age (Yrs.) & Female (\%) & AF & VT & PVC \\
\hline Von Olshausen, 1984 [6] & Germany & Prospective & DCM & 60 & $45 \pm 9$ & 10 & 11 & 25 & 60 \\
\hline Neri, $1996[5]$ & Italy & Prospective & $\mathrm{DCM}$ & 65 & $52 \pm 13$ & 20 & 11 & 29 & 62 \\
\hline Grimm, $2000[7]$ & Germany & Prospective & DCM & 202 & $50 \pm 13$ & 20 & 28 & 70 & - \\
\hline Grimm, $2003[8]$ & Germany & Prospective & $\mathrm{DCM}$ & 343 & $49 \pm 12$ & 22 & 80 & 111 & - \\
\hline Kamble, 2017 [9] & India & Prospective & $\mathrm{DCM}$ & 50 & - & 40 & 5 & - & - \\
\hline Kafle, 2018 [10] & Nepal & Retrospective & $\mathrm{DCM}$ & 400 & $57 \pm 13$ & 39 & - & - & - \\
\hline Kapoor, 2018 [11] & India & $\mathrm{X}$-sectional & DCM & 60 & $<40$ & 50 & 8 & 6 & - \\
\hline Saha, 2018 [12] & India & $\mathrm{X}$-sectional & DCM & 30 & $56 \pm 16$ & 43 & 4 & 3 & 14 \\
\hline Boyilla, 2019 [13] & India & Prospective & $\mathrm{DCM}$ & 60 & $57 \pm 16$ & 43 & 4 & 28 & 16 \\
\hline Savage, 1978 [14] & U.S. & Retrospective & $\mathrm{HCM}$ & 134 & - & 28 & 7 & - & - \\
\hline Corrado, 1998 [15] & Italy & Prospective & $\mathrm{HCM}$ & 49 & $23 \pm 7$ & 10 & - & 6 & - \\
\hline Elliott, 2000 [16] & U.K. & Prospective & $\mathrm{HCM}$ & 368 & $33 \pm 14$ & 35 & 10 & 64 & - \\
\hline Sachdev, 2002 [17] & U.K. & Prospective & $\mathrm{HCM}$ & 153 & - & 0 & - & 48 & - \\
\hline Dumont, 2006 [18] & Spain & Prospective & $\mathrm{HCM}$ & 102 & $51 \pm 14$ & 36 & 13 & 19 & - \\
\hline Adabag, 2008 [19] & U.S. & Prospective & $\mathrm{HCM}$ & 177 & $41 \pm 16$ & 27 & - & 64 & 136 \\
\hline McLeod, 2009 [20] & U.S. & Prospective & $\mathrm{HCM}$ & 2350 & $47 \pm 21$ & 44 & 424 & - & - \\
\hline Chin, 1990 [21] & U.S. & Prospective & LVNC & 8 & $9.04 \pm 7.24$ & 38 & - & 2 & - \\
\hline Oechslin, 2000 [22] & Switzerland & Prospective & LVNC & 34 & $42 \pm 17$ & 26 & 9 & 14 & - \\
\hline Murphy, 2005 [23] & U.K. & Prospective & LVNC & 45 & 37 & 38 & 3 & 10 & - \\
\hline Caliskan, 2011 [24] & Netherlands & Prospective & LVNC & 77 & $40 \pm 14$ & 52 & 7 & 17 & - \\
\hline Caliskan, 2012 [25] & Netherlands & Prospective & LVNC & 84 & $40(17-79)$ & 46 & - & 5 & - \\
\hline Brescia, 2013 [26] & U.S. & Retrospective & LVNC & 242 & $7.2 \pm 6.9$ & 40 & 4 & 42 & - \\
\hline Basso, 1996 [27] & Italy & Retrospective & ARVC & 30 & $28(15-65)$ & 33 & 2 & 15 & - \\
\hline Nava, $2000[28]$ & Italy & Prospective & ARVC & 138 & $33.7 \pm 14.5$ & 38 & - & 63 & 43 \\
\hline Peters, 2003 [29] & Germany & Prospective & ARVC & 265 & 46.8 & 40 & 6 & 132 & - \\
\hline Nasir, $2004[30]$ & U.S. & Prospective & ARVC & 50 & $38 \pm 15$ & 46 & - & 35 & 11 \\
\hline De Cobelli, 2006 [31] & Italy & Prospective & ARVC & 23 & $40.6 \pm 14.25$ & 35 & - & 14 & - \\
\hline Steriotis, 2009 [32] & Italy & Prospective & ARVC & 205 & $39 \pm 14$ & 35 & - & - & - \\
\hline Quarta, 2010 [33] & Italy & Prospective & ARVC & 68 & 43.8 & 51 & - & 30 & 32 \\
\hline Kamath, 2011 [34] & U.S. & Prospective & ARVC & 87 & $37 \pm 13$ & 46 & - & 50 & - \\
\hline Hoffmayer, 2011 [35] & U.S. & Retrospective & ARVC & 59 & - & - & - & 59 & 41 \\
\hline Sarvari, 2011 [36] & Norway & Prospective & ARVC & 69 & $43.7 \pm 15.9$ & 41 & - & 41 & - \\
\hline Benotti, 1980 [37] & U.S. & Prospective & $\mathrm{RCM}$ & 9 & 47 & 33 & 2 & - & - \\
\hline Falk, 1984 [38] & U.S. & Prospective & $\mathrm{RCM}$ & 27 & $58 \pm 11$ & 33 & 10 & 14 & 6 \\
\hline Ammash, 2000 [39] & U.S. & Retrospective & $\mathrm{RCM}$ & 94 & 64 & 61 & 70 & - & 13 \\
\hline Rahman, 2004 [40] & U.S. & Retrospective & $\mathrm{RCM}$ & 58 & 64 & 28 & 9 & - & - \\
\hline Hayashi, 2007 [41] & Japan & Retrospective & $\mathrm{RCM}$ & 12 & 4 & - & 1 & - & - \\
\hline Okamoto, 2011 [42] & Sweden & Prospective & $\mathrm{RCM}$ & 104 & 45 & 45 & 2 & 1 & - \\
\hline Cheng, 2013 [43] & China & Retrospective & $\mathrm{RCM}$ & 189 & $55 \pm 12$ & 39 & 30 & - & - \\
\hline Orini, 2019 [44] & U.K. & Prospective & $\mathrm{RCM}$ & 21 & $73 \pm 19$ & 10 & 3 & - & - \\
\hline
\end{tabular}

AF: Atrial Fibrillation; ARVC: Arrhythmogenic Right Ventricular Cardiomyopathy; DCM: Dilated Cardiomyopathy; LVNC: Left Ventricular Non-Compaction; PVC: Premature Ventricular Contractions; RCM: Restrictive Cardiomyopathy; VT: Ventricular Tachycardia 
In total, the 40 studies had a population size of 6,601 patients diagnosed with four structural types of CM (DCM, HCM, RCM, ARVC, and LVNC). The population included both children and adults, with significant age variations across the individual studies. On average the patient population was relatively young, with an average age of $42.7 \pm 15.3$ years. There was unequal representation of the sexes, with a male preponderance. About two thirds of the total patients $(65.3 \%)$ were males and about a third were females (34.7\%). However, gender representation varied significantly across the studies based on the type of the CM studied. For instance, the seven studies [14-20] recruiting HCM patients had the lowest proportion of females (25.7\%) and one study [17] assessing HCM patients recruited only male patients.

\section{Study findings}

Arrhythmias is a common presentation in patients suspected or confirmed with CM irrespective of the structural form of the disease. The most common forms of arrhythmias investigated are DCM, HCM, RCM, ARVC, and LVNC. The prevalence of arrhythmias varied across these five types of CM. However, there were no reports on the overall incidence of arrhythmias, only reports for the prevalence of specific types of arrhythmias. In addition, the most common types of arrhythmias among CM patients reported by the 40 studies are atrial fibrillation, ventricular tachycardia (VT) and premature ventricular contractions (PVC). The prevalence of these three different types of arrhythmias also varied across the different structural forms of the disease.
Atrial fibrillation: Among arrhythmias, AF remains the most common type of arrhythmias studied in CM patients, as well as in HF patients. In a pooled analysis of 27 studies [5-9,11-14,16,18,20,2224,26,37-44], 763 patients out of 5,031 presented with AF, translating into an overall prevalence of $12.8 \%$ (95\% confidence interval [CI] 9.9\% to $16.3 \%$ ] (Figure 1). The overall heterogeneity across the studies was high $\left(I^{2}=91 \%\right)$ and statistically significant $(\mathrm{p}=0.000)$. However, the high heterogeneity was due to differences in patient characteristics and ECG diagnostic method.

Figure 1 illustrates that the prevalence of AF varied across the different types of CM. Patients with RCM had the highest proportion of AF, affecting 127 out of a total of 514 patients reported in eight studies [37-44], translating into a prevalence of $19.2 \%$ (95\% CI: 7.3\% to $41.8 \%$ ).

The second highest prevalence rates occurred among patients with DCM assessed in eight studies [5-9,11-13]. On index presentation at the hospital, AF was present in 151 out of $870 \mathrm{HCM}$ patients, translating into a prevalence of $15.2 \%$ (95\% CI $11.4 \%$ to $20.1 \%$.

The prevalence of AF was much lower in HCM, ARVC and LVNC forms of CM. Among 2,954 HCM patients in four studies [14,16,18,20]. On index presentation, AF was detectable in 454, translating into a prevalence of $8.0 \%$ (95\% CI $3.1 \%$ to $19.0 \%$ ).

Patients with LVNC totalled to 398, out of which 23 tested for the presence of $\mathrm{AF}$, which is a prevalence pf $7.7 \%$ (95\% CI $2.3 \%$ to $22.9 \%$ )

Atrial Fibrillation: Event Rate and $95 \% \mathrm{Cl}$

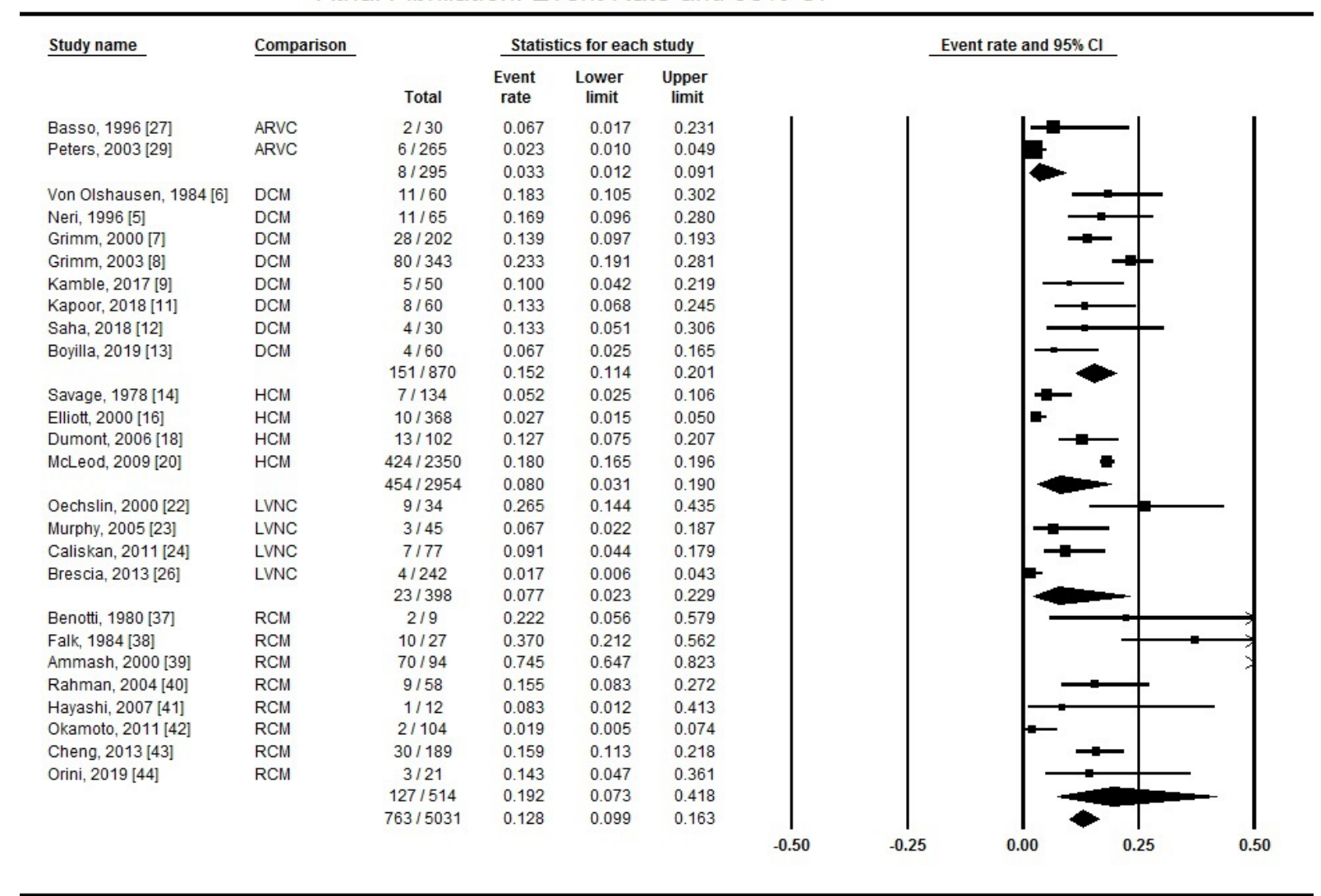

Heterogeneity: $Q=278.25 ; \operatorname{df}(Q)=25(p=0.000) ;$ Inconsistency $(I-S q u a r e d)=91.015 \%$

Figure 1. Forest plot showing atrial fibrillation event rate 
[22-24,26]. Finally, out of 295 ARVC patients reported in two studies [27,29], eight had AF, a prevalence of $3.3 \%$ (95\% CI $1.2 \%$ to $9.1 \%)$.

\section{Ventricular tachycardia}

Besides AF, VT is another common type of arrhythmia extensively studied among CM patients. Figure 2 summarizes the overall event rate of VT in all the CM patents as well as in different types of CM.

In 26 studies recruiting 3,079 patients, 1,017 had detectable AF, which translate into a high prevalence of $38.0 \%$ (95\% CI $33.5 \%$ to 42.7\%) [27-31,33-36,5-8,11-13,15-19,21-26, 38,42]. Heterogeneity across the studies was significantly high $\left(\mathrm{I}^{2}=90.44 \% ; \mathrm{p}=0.000\right)$ due to differences in patient population and ECG diagnostic methods across the included studies.

The presence of VT was the highest among patients with ARVC. Out of 789 ARVC patients in nine studies, 439 had detectable VT on admission on ECG, translating into a prevalence of $55.1 \%$ (95\% CI $47.8 \%$ to $62.3 \%$ ) [27-31,33-36].

Patients with DCM had the highest prevalence of VT on admission. Seven studies reported detectable VT on ECG on 272 patients out of 820 , which represents a prevalence of $32.4 \%$ (95\% CI $24.8 \%$ to $41.2 \%$ ) [5-8,11-13]. It means that about a third of DCM patients irrespective of the underlying aetiology have a clinical form of VT.
Similarly, the presence of VT on ECG was also common in patients with a clinical diagnosis of HCM. Five (5) studies reported VT on ECG in 201 patients out of 849 , which is a prevalence of $22.9 \%$ (95\% CI $15.4 \%$ to $32.8 \%$ ) [15-19].

Ventricular tachycardia was also prevalent among patients with a clinical diagnosis of LVNC. Six (6) studies recruiting 490 LVNC patients reported 90 had ECG evidence of VT, which is a prevalence of $20.2 \%$ (95\% CI $13.0 \%$ to $30.1 \%$ ) [21-26].

The prevalence of VT in the present analysis was the lowest among patients diagnosed with RCM. However, only two studies [38,42] reported VT in $131 \mathrm{RCM}$ patients, which may be inadequate to provide an accurate clinical picture. Of the 131 patients, 15 had detectable VT on ECG, a prevalence of $10.1 \%$ (95\% CI $0.1 \%$ to $9.2 \%$ ).

\section{Extrasystoles (PVCs)}

The third most commonly studied arrhythmias among CM patients is extrasystoles, which are premature contractions. Among the 40 included studies, PVC is the most extensively studied extrasystoles among patients with a clinical diagnosis of CM. Figure 3 shows that, in eleven studies recruiting 828 patients, 434 had detectable PVCs on admission on ECG, translating into a prevalence of $56.6 \%(95 \%$ CI $49.9 \%$ to $63.1 \%)[28,20,33,35,5,6,12,13,19,38,39]$. Similarly, the

Ventricular Tachycardia: Event Rate and 95\% Cl

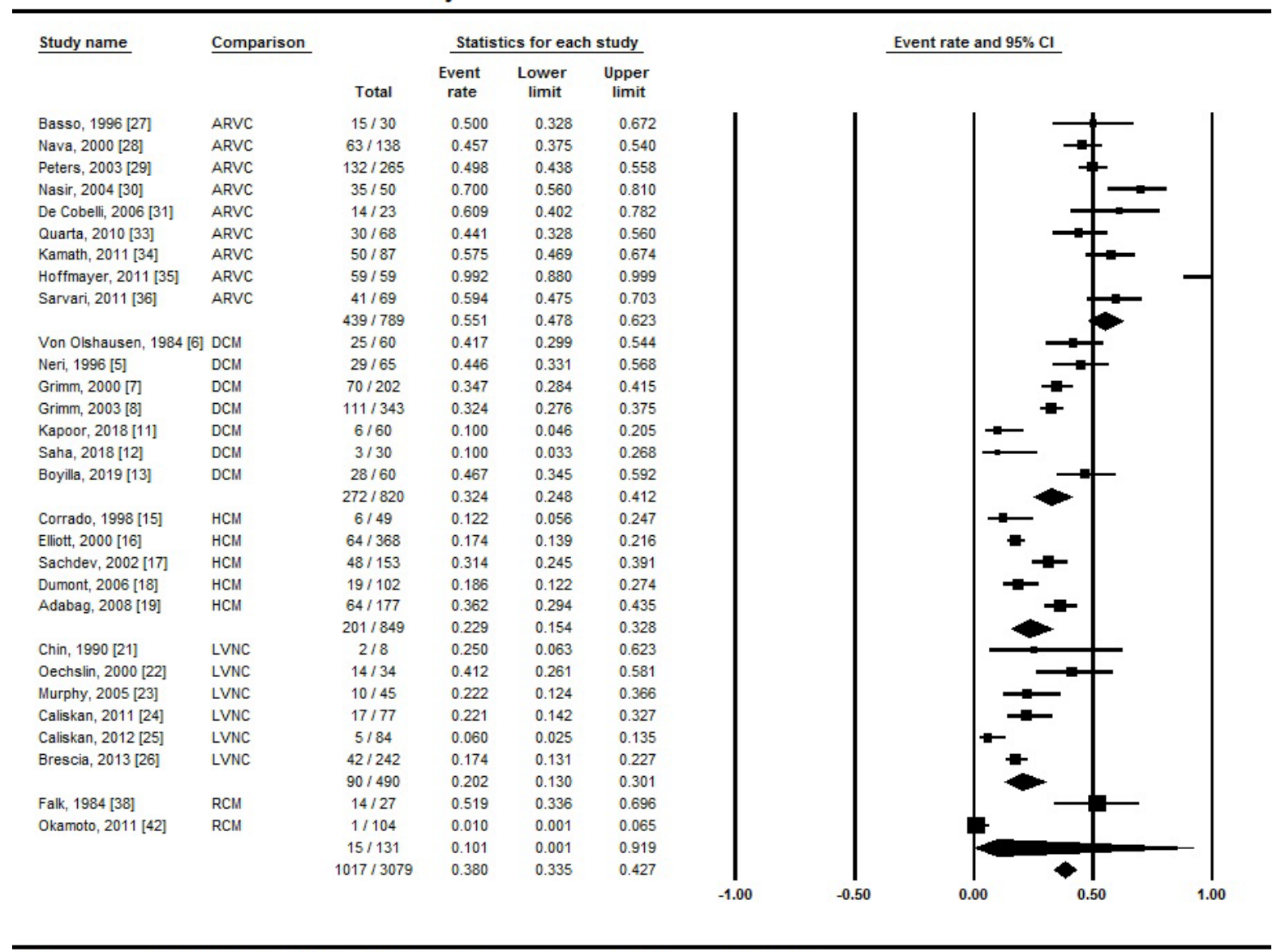

Heterogeneity: $Q=292.76 ; \operatorname{df}(Q)=28(p=0.000) ;$ Inconsistency $(I-S q u a r e d)=90.44 \%$

Figure 2. Forest plot showing ventricular tachycardia event rate 
Premature Ventricular Contractions: Event Rate and $95 \% \mathrm{Cl}$

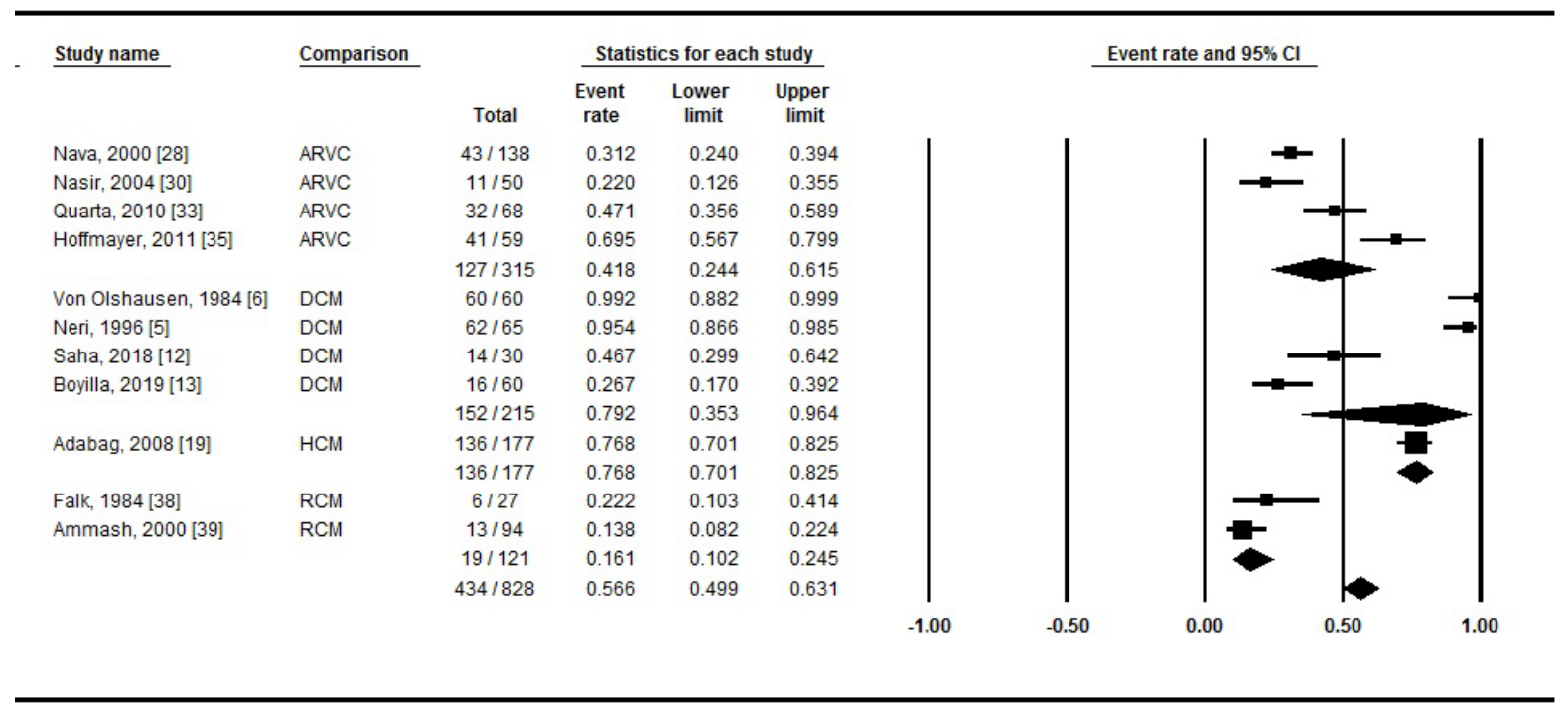

Heterogeneity: $Q=179.003 ; \mathrm{df}(Q)=10(p=0.000)$; Inconsistency $($ I-Squared $)=94.41 \%$

Figure 3. Forest plot showing premature ventricular contractions event rate

heterogeneity across the 11 studies was high $\left(\mathrm{I}^{2}=94.4 \% ; \mathrm{p}=0.000\right)$ associated with significant inter-study variability concerning patient characteristics, ECG diagnostic method and underlying aetiologies.

Patients with a clinical diagnosis of DCM had the highest proportion of PVCs. Four studies with a patient population of 215 DCM patients reported detectable PVC in 16 patients, representing a high prevalence of $79.2 \%$ (95 CI $35.3 \%$ to $96.4 \%$ ) $[5,6,12,13]$. However, the number of studies and the small sample size may not provide an accurate or reliable prevalence.

Premature ventricular contractions is also common among patients with ARVC. In four studies investigating 315 ARVC patients, 127 had PVCs on admission on ECG, a prevalence of $41.8 \%$ (95\% CI $24.2 \%$ to $61.5 \%)[26,30,33,35]$. The low number of studies and population size is inadequate to provide an accurate and reliable analysis.

Patients with RCM and HCM also had PVCs on index admission at the hospital but only two studies [38,39] and one study [19] provided the proportion of patients with PVCs. Few others reported extrasystoles in general, without mentioning the specific types. Out of 121 patients diagnosed with patients, 19 had PVCs on admission, a prevalence of $56.6 \%$ (95\% CI $10.2 \%$ to $24.5 \%$ ). The one study [19] investigating 177 HCM patients reported 136 had PVCs. However, the study investigated the prevalence and frequency of tachyarrhythmias with regard to delayed enhancement contrast-enhances cardiac MRI in HCM patients, which might have influence patient selection to those with a higher probability of having arrhythmias.

\section{Discussion}

\section{Arrhythmias}

Arrhythmias is a general medical term for abnormalities in the heart rhythm, where the heart is not in sinus rhythm (60-100 beats/minute in adults when resting). It occurs secondary to disruptions in the initiation or propagation of the electrical signals. Arrhythmias occur independent of the person's state (activity or emotions). Arrhythmias that start from the atria are supraventricular because they start supra (above) the ventricles and those start at the ventricles are ventricular arrhythmias. Arrhythmias that cause the heart to beat too fast are termed tachycardia ( $>100$ beats per minute in adults), or too slow are termed bradycardia ( $>60$ beats per minute in adults). Some arrhythmias cause fibrillation, where cardiac electrical signals results in an erratic and uncoordinated heartbeat. In patients with CM, a disorder of the heart muscle, have an increased risk of arrhythmias compared to the general population. Knowledge of the prevalence and types of arrhythmias in these patients is clinically relevant because of diagnostic, prognostic and therapeutic implications. The present meta-analysis finds that in CM patients, AF, VT and PVC are a common manifestation.

\section{Atrial fibrillation}

Atrial fibrillation (AF) is the most common arrhythmia encountered in clinical practice detectable by ECG, which is one of the recommended initial tests in patients suspected with CM. It is the most common and extensively studied type of arrhythmia due to its severe prognostic and therapeutic implications associated with an elevated risk of stroke, HF and other health-related complications [45]. It is a potential lethal form of supraventricular arrhythmias, which starts supra (above) the ventricles characterized by rapid, erratic and uncoordinated cardiac rhythm. Often, AF begins as brief periods of abnormal beating, which over time becomes longer and continuous. The presence of CM, a myocardial disorder, increases the risk of developing AF. Similarly, in the present study, the prevalence of AF in CM patients was high. The overall prevalence was $12.8 \%$. Patients with RCM exhibit the highest prevalence of AF on ECG at 19.2\%, followed by DCM patients at $15.2 \%$, HCM at $8.0 \%$, LVNC at $7.7 \%$ and finally ARVC at 3.3\%. The high prevalence of AF among RCM patients at index hospital admission is consistent with reports in published cardiology literature.

The high prevalence of AF in RCM patients is consistent with published literature and diagnostic and clinical management guidelines. The pathophysiology of RCM increases vulnerability towards supraventricular arrhythmias, especially AF. The ventricles become rigid due to the replacement of the normal myocardial tissues with 
scarred tissues. The result is reduced elasticity of myocardial tissues leading to abnormal relaxation and reduced ability of the ventricles to fill with blood resulting in enlarged atria and consequently cardiac circulation is reduced over time. These changes lead to increased risk of AF and consequently asymptomatic HF $[46,47]$. In addition, RCM tends to affect older adults. Since the risk of AF increase with age, RCM patients may show a higher prevalence of $\mathrm{AF}$ relative to other structural forms of CM [48]. In a recent review of RCM, Muchtar et al. [48] reports a variable prevalence of AF due to different underlying aetiology. RCM due to wild-type transthyretin (ATTRwt) amyloidosis has the highest prevalence of $\mathrm{AF}$ at $45 \%$ followed by RCM due to mutant transthyretin (ATTRm) at $15 \%$ and RCM due to light chain immunoglobulin (AL) amyloidosis at $12 \%$. The current meta-analysis did not evaluate different aetiologies of RCM and reported the overall prevalence of $19.2 \%$, which is consistent with the review by Muchtar et al. [49]. However, the presence of AF in RCM patients is not associated with increased mortality but linked to the development of asymptomatic HF [47,48].

Besides RCM, DCM patients are more likely to develop AF relative to other structural forms of CM. The present meta-analysis finds the prevalence of AF in patients diagnosed with DCM at index presentation is $15.2 \%$. In a recent study in Egypt recruiting 50 DCM patients, consisting of non-ischemic $(n=20)$ and ischemic aetiologies $(n=30)$, the prevalence was $15.0 \%$ for non-ischemic and $53.3 \%$ for ischemic aetiologies, indicating ischemic DCM has a much higher prevalence for $\mathrm{AF}$ that non-ischemic causes The findings are consistent with the present findings of $15.2 \%$ among DCM patients of non-ischemic aetiologies [50]. The high prevalence of AF among DCM patients has been linked to its pathophysiology that increases susceptibility to AF. Patients with DCM exhibit a disruption of the link between the sarcolemma, the cytoskeleton and the sarcomere, and the final common pathway of arrhythmias results from the disturbance of ion channel function [5154]. Thus, since the majority of dysfunctional ion channels occur in the sarcolemma, the disruption of sarcolemma-sarcomere link could lead to ion channel dysfunction to cause a combination of DCM and arrhythmias [3]. In DCM patients, AF in the setting of LV dysfunction increases mortality and morbidity risk.

The present analysis reported AF prevalence of $8 \%$ among HCM patients. However, recent studies that exclusively focused on AF in HCM patients report a much higher prevalence. Rowin et al. [55] studied 1,558 consecutive patients and reported $20 \%$ lifetime prevalence consisting of $74 \%$ symptomatic paroxysmal AF and $26 \%$ permanent AF preceded by paroxysmal AF episodes and as high as $40 \%$ in HCM patients 70 years or older. In a related study, Siontis et al. [56] finds a prevalence of $\mathrm{AF}$ among 3,673 referral patients at $18 \%$, disproportionately affecting older adults and those with non-obstructive HCM with larger left atria, higher E/e ratios and worse cardiopulmonary exercise. The prevalence in a large referral HCM population, AF affect about 1 in every five patients. Robinson et al. [57] investigated 52 consecutive HCM patients and associated the presence of $\mathrm{AF}$ with worsening symptoms in $89 \%$ of the patients. Duration of arrhythmia and amiodarone therapy were the most powerful predictor of return to sinus rhythm. The cause of AF in HCM patients remains unclear. However, evidence from small cohort studies associated old age and left ventricular outflow tract (LVOT) with increased risk of AF. Echocardiographic markers of left atrial (LA) dysfunction such as LV diameter, volume index, fractional shortening and ECG abnormalities such P-wave dispersion show a correlation with $\mathrm{AF}$ in $\mathrm{HC}$ patients, but the evidence have not been validated by large cohort studies.

In the present analysis, the average AF prevalence in LVNC patients was $7.7 \%$. The findings were based on a small sample of 398
LVNC patients from four small cohort studies [22-25]. An earlier study, Ritter et al. [58] reported a low prevalence of AF among LVNC patients ranging between $0.01 \%$ and $0.04 \%$. However, with improvement in cardiac imaging modalities and consequently the diagnosis of LVNC, the prevalence of AF has increased significantly over time, and it is the most prevalent atrial arrhythmia in LVNC patients. However, in major reports, the incidence of AF in LVNC ranges between $5 \%$ and $29 \%$ depending on the age of the cohort. The incidence is significantly higher in children since it is the most common primary CM but cases of LVNC are increasing in adults because of improved imaging modalities. In patients with transthoracic echocardiographic evidence of LVNC, $28 \%$ had AF whose average age was $53 \pm 17$ [59].

Pathophysiologically, LVNC is the consequence of the failure in cardiac embryogenesis, which leads to unusual trabeculations in the LV cavity which are most prominent in the mid-lateral-inferior parts of the LV. Trabeculated ventricle can serve as foci for arrhythmias, which is mostly atrial but can also be ventricular [58]. The ratio of noncompacted and compacted ventricles $>2.0$ and end diastolic epicardium to trough, to epicardium to peak of trabeculation (X:Y) ratio have no correlation with atrial arrhythmias but end systolic maximum noncompacted thickness has some association with atrial arrhythmias. A further multivariate analysis shows that LA volume index and maximum end systolic NC were associated with atrial arrhythmias [59].

Finally, of the primary CMs, ARVC have the least prevalence of 3.3\% as per the present findings. The prevalence was based on a small cohort of 295 patients reported in two studies $[27,29]$. Overall, the information on atrial arrhythmias in ARVC patients is limited and prevalence may vary significantly based on disease severity. In a study of 36 patients who satisfied task force criteria for ARVC and had a history of VT, Chu et al. [60] observes $42 \%$ had documented atrial arrhythmias - atrial flutter (11), AF (11), and atrial flutter together with AF (7). However, $97 \%$ of these patients had implantable cardioverter-defibrillator (ICD) devices, and 15 had atrial leads, explaining the high AF prevalence. $\mathrm{AF}$ is an arrhythmic manifestation of ARVC and an indicator of atrial involvement in disease progression. The prevalence correlates with ARVC diagnostic score - considerably increasing with a diagnostic score of 4 and increases further with increased diagnostic score [61].

The pathophysiologic mechanisms of AF in ARVC remains unclear. However, hemodynamic consequences of abnormalities in the RV contractility may contribute to right atrial (RA) overload, increased stretch and development of fibrosis in the atrial walls. The involvement of the LV leading to systolic dysfunction may also be associated with complications such as AF and thromboembolic events [62]. The development of atrial myocardial substrate in ARVC may be influenced by genetically determined desmosomal dysfunction [61]. AF may also result from the same mechanisms responsible for fibrotic replacement of the myocardium in the RV walls and occurs in parallel with the involvement of the ventricular myocardium $[61,62]$.

\section{Ventricular tachycardia}

In the present analysis, VT is the second most studied arrhythmia after AF. Same to AF, VT has important prognostic, diagnostic and therapeutic implications, which explains the research interest. The overall prevalence of VT in CM patients is $38 \%$, which is significantly higher than AF. Sustained VT is a leading cause of death in CM patients. Ventricular tachyarrhythmias, which include life threatening episodes of sustained VT and VF. Sustained VT and VF develop in the setting of acute myocardial infarction (MI) or as a clinical sequelae of advanced CM. MI may have a scar tissue surrounded by islands is viable 
myocardium. These islands slow down electrical conduction, which can exist the island creating a QRS complex and then re-enter the island, enabling VT to propagate. Catheter ablation prevents the re-entrant circuits and prevents the occurrence of future VTs [63].

The exact prevalence of VTs in CM patients are lacking due to difference diagnosis approaches and the different phases of the disease. However, earlier retrospective and observational studies and registries of sudden cardiac death (SCD) suggested a high prevalence. However, the over the few past decades, there has been a gradual decline in the incidence of lethal VTs due to upstream treatment for and prevention of coronary artery disease (CAD) and its sequelae. In addition, primary prevention ICD (the mainstay treatment for VT) have improved survival in patients at risk of VTs [64]. However, ICD therapy cannot prevent future arrhythmias, it is painful, leads to psychological distress and higher mortality relative to patients without ICD therapy. Catheter ablation is considered an alternative to medical; therapy in patient with refractory VT [64]. Despite the reduced prevalence of VT among CM patients, it differs across structural forms of CMs - DCM, HCM, ECM, ARVC and LVNC.

Patients with ARVC have the highest prevalence of VT with slightly more than a half $(55.1 \%)$ affected. However, this prevalence may be much lower than the true prevalence because the findings were based on a small sample and the lack of determination of what stage the disease was - early silent phase, unstable phase with symptomatic arrhythmias, $\mathrm{RV}$ failure phase and final phase with progressive biventricular dilation [65]. The high prevalence of VT among ARVC patients is related to the traditional thinking that $\mathrm{CM}$ with the involvement of $\mathrm{LV}$ and ischemic heart disease were the primary causes of VAs and SCD. However, over the past two decades, VAs originating from the RV have attracted growing attention of scientist because they disproportionately affect younger patients associated with an increased risk of SCD [66].

The reported prevalence of $55.5 \%$ is consistent with research history of ARVC. Initially, the prevalence of VTs in ARVCs was believed to be $100 \%$. As recently as the 1980 s, ventricular arrhythmias were considered the presenting symptoms of ARVC [67]. In the first series of ARVC patients by Marcus et al. [68] in 1982, reported all patients presented with VAs. However, over time, with improvement in diagnostics, registry data allowed valuable insight into less advanced forms of the ARVC phenotype. In a recent North American Multidisciplinary study [69], which categorized patients into 108 probands with suspected ARVC, VT were not universal in all patients. Sustained VT was present in $35 \%$ of 75 patients who underwent electrophysiology study and sustained VT was inducible during an electrophysiological study in $49 \%$. Among patients with sustained VT, the most common ECG finding was morphology of LBBB/superior axis (36.8\%), LBBB/Inferior axis $(26.3 \%)$, indeterminate morphology $(13 \%)$, and RBBB pattern (2.6\%) [69].

The available evidence suggests that among ARVC patients, VTs are a common presentation and an important ECG-based diagnostic clue in the presence of progressive structural abnormalities of the RV. Myocardial degeneration may extend to the LV, especially in the advance stages of the disease. The hallmark of ARVC, massive or partial fibrofatty replacement of the normal tissues of the myocardium may provide a substrate for electrical instability leading to VAs ranging from isolated PVC to sustained VT or VF [67]. Among ARVC patients, VT present an important ECG feature for a differential diagnosis of VT from the RV outflow tract and ARVC, which is important because or the benign nature of the former and the need for sudden cardiac risk stratification and family screening in the latter [70].
ECG-assessed VT is also common in DCM patients, generally affecting about a third of the patients (32.4\%). These findings are in support to an earlier report by Zecchin et al. [71] of a prevalence of $40 \%$ of non-sustained VT among DCM patients, which can be much higher in familial forms such as lamin A/C mutations. The high prevalence of VT among DCM patients is consistent with reports from an earlier meta-analysis. Sammani et al. [72] performed a meta-analysis of 28 studies predicting arrhythmic risk in non-ischemic DCM patients. In these patients, the risk of life threatening VT was $4.3 \%$ per year and much higher in patients with co-existing hypertension, a history of life-threatening VT, decreased LVEF, high LVEDE/LVESV, a history of non-sustained VT, and delayed gadolinium enhancement. Some patients with DCM presenting with ventricular arrhythmias early in the course of the disease unrelated to the severity of the disease may have an arrhythmogenic phenotype [73]. Thus, a family history of VAs in DCM predict an ominous prognosis and increased risk of SCD.

Multiple mechanisms can explain the pathogenesis of VTs in DCM patients but the most common is re-entry electrical circuits associated with slow conduction across viable muscle bundles within regions of interstitial fibrosis. Other possible mechanisms include non-uniform anisotropy of impulse propagation, ion channel dysfunction, and reduced gap junction function [72]. Despite developing knowledge in the pathogenesis of VTs, the prognostic significance of VTs in DCM patients is unclear and conflicting data has been published in the past 30 decades. Although the rates of SCD have declined over the past 30 years, identification of patients at risk remains to be elucidated. However, the LV dysfunction is pivotal for the identification of candidates for ICD implantation based on the current guidelines, while the role of other invasive and non-invasive parameters remains debatable. Cardiac magnetic resonance imaging and genetic analysis may prove useful in identification of at risk patients but they are not widely available. Unlike ARVC, in DCM patients, VTs can be the consequence as well as the cause of DCM in the presence of sustained heart rate $>100 /$ minute after the exclusion of all other known causes.

In HCM patients, the present analysis finds that VT affects $22.9 \%$ of the patients, meaning about 1 in every $10 \mathrm{HCM}$ patients may present with VT. Although, the exact prevalence of VTs in HCM has not been determined, it has been associated with increased risk of SCD in this cohort. Approximately between $60 \%$ and $70 \%$ of HCM patients die suddenly due to cardiac causes with ventricular tachyarrhythmias implicated as the main cause [19]. The high arrhythmic propensity in HCM patients may result from a combination of primary substrate abnormalities including hypertrophy, myocardial fibre disarray, interstitial fibrosis, and secondary triggers such as ischemia, physical exercise, and excessive sympathetic stimulation. Such heterogeneity in substrate and triggers considerably reduce the predictive accuracy of risk stratification model [74]. In addition, study population have a low event rate and effect of medical therapy limits the application of predictive models for VTs in HCM patients.

New insights into the pathogenic mechanism of HCM has improved the understanding of the risk of developing lethal arrhythmias. Nonsustained VT detected on Holter monitoring in younger patients ( $<39$ years) causes a higher mortality rate [75] However, in older patients, cardiomyocyte loss and fibrosis contributes incrementally to VT development, and thus, the increased mortality risk in younger patients suggests a more potent arrhythmogenic substrate secondary to cardiomyocyte disarray, myocardial ischemia and abnormal autonomic function. Although the association between SCD and non-sustained VT has been documented, the majority of SCDs in HCM patients occur 
in patients without non-sustained VT, which demonstrates that Holter monitoring only detects a subset of HCM subjects at a higher risk of VT [74]. Despite the role of 12-lead ECG in the detection of arrhythmias in HCM patients, it play no role in predicting arrhythmia risk since none of the ECG parameters cannot predict candidates for ICD for VTs and not useful for risk stratification [76].

In LVNC patients, the prevalence of VTs is $20.2 \%$ but was based on a small sample. However, the true prevalence of VT in LVNC patients has not been well described because of a very low prevalence of the LVNC. In a large hospital cohort together with a ten-year experience, Ritter et al. [77] reported a prevalence of $0.05 \%$ despite an increased awareness of the LVNC phenotype. Different patient population and different stages of the disease, also contribute to complicate the determination of prevalence. LVNC may increase the risk of VT and the risk of thromboembolism, possibly due to reduced blood flow in the intertrabecular recesses [78]. The role of ECG in the diagnosis of LVNC patients or for risk stratification has not been defined possible due to the overall low prevalence of the phenotype in CM.

Finally, RCM has the least prevalence of VTs of $10.01 \%$ based on a small sample of 131 from only two studies. However, very little is known about arrhythmias in RCM. It is the least common primary type of CM as well as has variable pathogenesis, clinical presentation, diagnostic evaluation and criteria, treatment and prognosis, which complicates the study of prevalence problematic [79]. Pathophysiologically, RCM results from increased myocardial stiffness leading to impaired ventricular filling. Biventricular chamber size and systolic function are usually normal or near normal until the advanced stages of the disease. It affects one or both ventricles and may cause signs and symptoms of left or right HF with frequent encounters of arrhythmias and conduction disturbances [49]. Additional controlled trials are warranted to determine the prevalence and frequency of VTs in RCM patients.

\section{Premature ventricular contractions}

Premature contraction or extrasystoles are a common manifestation in patients with a clinical diagnosis of CM. It is an extra beat or contraction that interrupts the sinus rhythm independent of the sinus rhythm arising secondary to an impulse in the part of the heart other than the Sino-atrial node (SAN), followed by a pause, as the cardiac electrical system resets itself and the contraction following is usually more forceful. In the present analysis, the overall prevalence of PVCs in CM patients was $56.6 \%$. The high prevalence is consistent with reports in literature that PVCs affect $1 \%$ and $40 \%$ to $75 \%$ of heathy people assessed by the standard ECG and 24 to 48 hours ambulatory ECG recording [80]. The prevalence is much higher (up to 90\%) in patients with structural heart diseases, especially CM. PVCs are the most common type of arrhythmia secondary to myocardial infarction, as well as among patients with severe LV hypertrophy, HCM and congestive HF [81]. They are clinically relevant because PVCs could potentially lead to a deterioration of the systolic function in non-ischemic CM or result in PVC-induced CM. They could also provide important prognosis information. Thus, in these patients, ambulatory monitoring is recommended to assess for PVC burden, especially in ARVC and HCM phenotypes. The same mechanisms that trigger VT also cause PVCs, in which case, catheter ablation is the mainstay treatment [81].

PVCs in DCM patients have a very high prevalence of $79.2 \%$. Studies on arrhythmias in the DCM phenotype have largely focused on $\mathrm{AF}$ and VT because of increased risk of death and the need for specific anticoagulation and catheter ablation respectively [72]. However, the high prevalence among DCM patients may be associated with a high PVC burden above $24 \%$ can induce or contribute to development of PVC-induced DCM and HF [82]. In patients with ARVC, about two in every five (41\%) present with PVCs. However, the prevalence of PVCs in ARVC patients remains not well documented since the majority of studies appear to focus on VTs with a mention of PVCs in commentary. The focus stems from the observation that ventricular arrhythmias is a major characteristic of ARVC patients, but it may progress to VF accompanied by PVCs. In addition, PVCs share the same pathogenetic mechanisms of VTs, which mainly includes re-entry circuits [67]. Further, in ARVC patients, the 24-hour ECG monitoring can detect $89.5 \%$ to refine grouping based on the 2010 Task Force Criteria [83]. In the other primary forms of CM, RCM and HCM, the prevalence of PVCs could not be pooled because of insufficient data. The lack of studies on PVCs in these CM phenotypes also suggest the impact of PVCs on patient health and treatment may be relatively less significant and possible explains educed research interest.

\section{Clinical implications}

ECG tests are important initial tests in patients with CM. In addition to the assessment of electrical conduction, ECG tests are important in the detection of arrhythmias. The detection of arrhythmias such as AF, VT and PVC is clinically relevant since the three can be life-threatening and may require treatment modification to include anticoagulation therapy for $\mathrm{AF}$, and catheter ablation or ICD for VT and PVCs. In particular, ECG is an important diagnostic test in DCM and DCM patients for AF and VTs, and VT and PVCs for ARVC patients. In ARVC patients, ECG is an important differential diagnostic test because of its ability to distinguish idiopathic VTs from VTs or PVCs due to ARVC and RVOT.

\section{Conclusion}

ECG-detected arrhythmias are common clinical manifestations in patients with CM. However, AF, VT, and PVCs are the most commonly studied in CM patients because of their diagnostic, prognostic, and therapeutic implications. Among arrhythmias, AF is more common in RCM and DCM whereas VTs and PVCs are more common among ARVC patients, as well as in DCM, HCM, and LVNC. The detection of $\mathrm{AF}$ is important because of associated increased risk of thromboembolic events and heart failure, and VT and PVCs for increased risk of SCD. Thus, ECG is an important diagnostic tool for the detection of arrhythmias, guiding therapy and differential diagnosis for ARVC patients from RVOT- associated VT.

\section{References}

1. Kurbel S (2014) A vector-free ECG interpretation with P, QRS \& T waves as unbalanced transitions between stable configurations of the heart electric field during PR, ST \& TP segments. Theor Biol Med Model 11: 10. [Crossref]

2. Su L, Borov S, Zrenner B (2013) 12-lead Holter electrocardiography. Herzschrittmacherther Elektrophysiol 24: 92-96. [Crossref]

3. Towbin JA, Lorts A (2011) Arrhythmias and dilated cardiomyopathy: Common pathogenetic pathways? J Am Coll Cardiol 57: 2169-2171. [Crossref]

4. Aziz T, Burgess M, Rahman AN, Campbell CS, Yonan N (2001) Cardiac transplantation for cardiomyopathy and ischemic heart disease: differences in outcome up to 10 years. $J$ Heart Lung Transplant 20: 525-533. [Crossref]

5. Neri R, Mestroni L, Salvi A, Camerini F (1986) Arrhythmias in dilated cardiomyopathy Postgrad Med J 62: 593-597. [Crossref]

6. Von Olshausen K, Schafer A, Mehmel HC, Schwarz F, Senges J, et al. (1984) Ventricular arrhythmias in idiopathic dilated cardiomyopathy. Br Heart J 51: 195-201. [Crossref] 
7. Grimm W, Glaveris C, Hoffmann J, Menz V, Muller HH, et al. (2000) Arrhythmia risk stratification in idiopathic dilated cardiomyopathy based on echocardiography and 12 lead, signal-averaged, and 24-hour Holter electrocardiography. Am Heart J 140: 43-51. [Crossref]

8. Grimm W, Christ M, Bach J, Muller HH, Maisch B (2003) Noninvasive arrhythmia risk stratification in idiopathic dilated cardiomyopathy: results of the Marburg Cardiomyopathy Study. Circulation 108: 2883-2891. [Crossref]

9. Kamble SD, Bobade RR (2017) Electrocardiographic and echocardiographic characteristics of patients with dilated cardiomyopathy. MedPulse International Journal of Medicine 3: 33-37.

10. Kafle RC, Paudel N, Sharma D, Alurkar VM (2018) Electrocardiographic profile of dilated cardiomyopathy in patients attending a tertiary care hospital of Western Nepal. Journal of Universal College of Medical Sciences 6: 40-45.

11. Kapoor S, Amin B, Jha RK (2018) A study of clinical profile of patients with dilated cardiomyopathy in correlation with ECG and echocardiography in age less than 40 years. Journal of Dental and Medical Sciences 17: 42-47.

12. Saha KK, Kumar A, Sneha K, Kumar P, Mishra AK, et al. (2018) A clinical study of dilated cardiomyopathy with correlation to electrocardiography and echocardiography: A cross sectional study. International Journal of Scientific Study 5: 91-95.

13. Boyilla V, Mogarala RR (2019) A study of clinical profile of dilated cardiomyopathy in correlation with ECG and echocardiography. Journal of Dental and Medical Science 18: $56-60$.

14. Savage DD, Seides SF, Clark CE, Henry WL, Maron BJ, etal. (1978) Electrocardiographic findings in patients with obstructive and nonobstructive hypertrophic cardiomyopathy. Circulation 58: 402-408. [Crossref]

15. Corrado D, Basso C, Schiavon, M, Thiene G (1998) Screening for hypertrophic cardiomyopathy in young athletes. $N$ Engl J Med 339: 364-369. [Crossref]

16. Elliott PM, Poloniecki J, Dickie S, Sharma S, Monserrat L, et al. (2000) Sudden death in hypertrophic cardiomyopathy: identification of high risk patients. $J$ Am Coll Cardiol 36: 2212-2218. [Crossref]

17. Sachdev B, Takenaka T, Teraguchi H, Tei C, Lee P, et al. (2002) Prevalence of Anderson-Fabry disease in male patients with late onset hypertrophic cardiomyopathy. Circulation 105: 1407-1411. [Crossref]

18. Dumont CA, Monserrat L, Soler R, Rodríguez E, Fernandez X, et al. (2006) Interpretation of electrocardiographic abnormalities in hypertrophic cardiomyopathy with cardiac magnetic resonance. Eur Heart J 27: 1725-1731. [Crossref]

19. Adabag AS, Maron BJ, Appelbaum E, Harrigan CJ, Buros JL, et al. (2008). Occurrence and frequency of arrhythmias in hypertrophic cardiomyopathy in relation to delayed enhancement on cardiovascular magnetic resonance J Am Coll Cardiol 51: 1369-1374. [Crossref]

20. McLeod CJ, Ackerman MJ, Nishimura RA, Tajik AJ, Gersh BJ, et al. (2009) Outcome of patients with hypertrophic cardiomyopathy and a normal electrocardiogram. $J \mathrm{Am}$ Coll Cardiol 54: 229-233. [Crossref]

21. Chin TK, Perloff JK, Williams RG, Jue K, Mohrmann R (1990) Isolated noncompaction of left ventricular myocardium. A study of eight cases. Circulation 82: 507-513. [Crossref]

22. Oechslin EN, Jost CHA, Rojas JR, Kaufmann PA, Jenni R (2000) Long-term follow-up of 34 adults with isolated left ventricular noncompaction: a distinct cardiomyopathy with poor prognosis. J Am Coll Cardiol 36: 493-500. [Crossref]

23. Murphy RT, Thaman R, Blanes JG, Ward D, Sevdalis E, et al. (2005) Natural history and familial characteristics of isolated left ventricular non-compaction. Eur Heart J 26: 187-192. [Crossref]

24. Caliskan K, Szili-Torok T, Theuns DA, Kardos A, Geleijns ML, et al. (2011) Indications and outcome of implantable cardioverter-defibrillators for primary and secondary prophylaxis in patients with noncompaction cardiomyopathy. J Cardiovasc Electrophysiol 22: 898-904. [Crossref]

25. Caliskan K, Ujvari B, Bauernfeind T, Theuns DA, Van Domburg RT, et al. (2012) The prevalence of early repolarization in patients with noncompaction cardiomyopathy presenting with malignant ventricular arrhythmias. J Cardiovasc Electrophysiol 23: 938-944. [Crossref]

26. Brescia ST, Rossano JW, Pignatelli R, Jefferies JL, Price JF, et al. (2013) Mortality and sudden death in pediatric left ventricular noncompaction in a tertiary referral center. Circulation 127: 2202-2208. [Crossref]

27. Basso C, Thiene G, Corrado D, Angelini A, Nava A, et al. (1996) Arrhythmogenic right ventricular cardiomyopathy: dysplasia, dystrophy, or myocarditis? Circulation 94 : 983-991. [Crossref]
28. Nava A, Folino AF, Bauce B, Turrini P, Buja GF, et al. (2000) Signal-averaged electrocardiogram in patients with arrhythmogenic right ventricular cardiomyopathy and ventricular arrhythmias. Eur Heart J 21: 58-65. [Crossref]

29. Peters S, Trümmel M (2003) Diagnosis of arrhythmogenic right ventricular dysplasiacardiomyopathy: value of standard ECG revisited. Ann Noninvasive Electrocardiol 8 238-245.

30. Nasir K, Bomma C, Tandri H, Roguin A, Dalal D, et al. (2004) Electrocardiographic features of arrhythmogenic right ventricular dysplasia/cardiomyopathy according to disease severity: a need to broaden diagnostic criteria. Circulation 110: 1527-1534 [Crossref]

31. De Cobelli F, Pieroni M, Esposito A, Chimenti C, Belloni E, et al. (2006) Delayed gadolinium-enhanced cardiac magnetic resonance in patients with chronic myocarditis presenting with heart failure or recurrent arrhythmias. J Am Coll Cardiol 47: 16491654. [Crossref]

32. Steriotis A K, Bauce B, Daliento L, Rigato I, Mazzotti E, et al. (2009) Electrocardiographic pattern in arrhythmogenic right ventricular cardiomyopathy. $\mathrm{Am}$ J Cardiol 103: 1302-1308. [Crossref]

33. Quarta G, Ward D, Esteban MTT, Pantazis A, Elliott PM, et al. (2010) Dynamic electrocardiographic changes in patients with arrhythmogenic right ventricular cardiomyopathy. Heart 96: 516-522. [Crossref]

34. Kamath GS, Zareba W, Delaney J, Koneru JN, McKenna W, et al. (2011) Value of the signal-averaged electrocardiogram in arrhythmogenic right ventricular cardiomyopathy/ dysplasia. Heart Rhythm 8: 256-262. [Crossref]

35. Hoffmayer KS, Machado ON, Marcus GM, Yang Y, Johnson CJ, et al. (2011) Electrocardiographic comparison of ventricular arrhythmias in patients with arrhythmogenic right ventricular cardiomyopathy and right ventricular outflow tract tachycardia. J Am Coll Cardiol 58: 831-838. [Crossref]

36. Sarvari SI, Haugaa KH, Anfinsen OG, Leren TP, Smiseth OA, et al. (2011) Right ventricular mechanical dispersion is related to malignant arrhythmias: a study of patients with arrhythmogenic right ventricular cardiomyopathy and subclinical right ventricular dysfunction. Eur Heart J 32: 1089-1096. [Crossref]

37. Benotti JR, Grossman W, Cohn PF (1980) Clinical profile of restrictive cardiomyopathy. Circulation 61: 1206-1212. [Crossref]

38. Falk RH, Rubinow A, Cohen AS (1984) Cardiac arrhythmias in systemic amyloidosis: correlation with echocardiographic abnormalities. J Am Coll Cardiol 3: 107-113. [Crossref]

39. Ammash NM, Seward JB, Bailey KR, Edwards WD, Tajik AJ (2000) Clinical profile and outcome of idiopathic restrictive cardiomyopathy. Circulation 101: 2490-2496. [Crossref]

40. Rahman JE, Helou EF, Gelzer-Bell R, Thompson RE, Kuo C, et al. (2004) Noninvasive diagnosis of biopsy-proven cardiac amyloidosis. J Am Coll Cardiol 43: 410-415. [Crossref]

41. Hayashi T, Tsuda E, Kurosaki K, Ueda H, Yamada O, et al. (2007) Electrocardiographic and clinical characteristics of idiopathic restrictive cardiomyopathy in children. Circ J 71: 1534-1539. [Crossref]

42. Okamoto S, Hörnsten R, Obayashi K, Wijayatunga P, Suhr OB (2011) Continuous development of arrhythmia is observed in Swedish transplant patients with familial amyloidotic polyneuropathy (amyloidogenic transthyretin Val30Met variant). Liver Transpl 17: 122-128. [Crossref]

43. Cheng Z, Zhu K, Tian Z, Zhao D, Cui Q, et al. (2013) The findings of electrocardiography in patients with cardiac amyloidosis. Ann Noninvasive Electrocardiol 18: 157-162. [Crossref]

44. Orini M, Graham AJ, Martinez-Naharro A, Andrews CM, de Marvao A, et al. (2019) Noninvasive mapping of the electrophysiological substrate in cardiac amyloidosis and its relationship to structural abnormalities. J Am Heart Assoc 8: e012097. [Crossref]

45. Bozkurt B, Colvin M, Cook J, Cooper LT, Deswal A, et al. (2016) Current diagnostic and treatment strategies for specific dilated cardiomyopathies: a scientific statement from the American Heart Association. Circulation 134: e579-e646. [Crossref]

46. Elliott P, Andersson B, Arbustini E, Bilinska Z, Cecchi F, et al. (2008) Classification of the cardiomyopathies: a position statement from the European Society Of Cardiology Working Group on Myocardial and Pericardial Diseases. Eur Heart J 29: 270-276. [Crossref]

47. Maron BJ, Towbin JA, Thiene G, Antzelevitch C, Corrado D, et al. (2006) Contemporary definitions and classification of the cardiomyopathies. Circulation 113: 1807-1816. [Crossref] 
48. Mirza M, Strunets A, Shen WK, Jahangir A (2012) Mechanisms of arrhythmias and conduction disorders in older adults. Clin Geriatr Med 28: 555-573. [Crossref]

49. Muchtar E, Blauwet LA, Gertz MA (2017) Restrictive cardiomyopathy: genetics, pathogenesis, clinical manifestations, diagnosis, and therapy. Circ Res 121: 819-837. [Crossref]

50. Kamal AM, Omara AA, Samy NI, AlAraby KM (2018) Incidence of atrial fibrillation in ischemic and nonischemic dilated cardiomyopathy. Menoufia Medical Journal 31: 387-391.

51. Bowles NE, Bowles KR, Towbin JA (2000) The "final common pathway" hypothesis and inherited cardiovascular disease: the role of cytoskeletal proteins in dilated cardiomyopathy. Herz 25: 168-175. [Crossref]

52. Towbin JA (2000) Cardiac arrhythmias: the genetic connection. $J$ Cardiac Electrophysiol 11: 601-602. [Crossref]

53. Vatta M, Marcus FI, Towbin JA (2007) Arrhythmogenic right ventricular cardiomyopathy: a "final common pathway" that defines clinical phenotype. Eur Heart $J$ 28: 529-530. [Crossref]

54. Towbin JA, Vatta M (2007) Myocardial infarction, viral infection, and the cytoskeleton: final common pathways of a common disease? J Am Coll Cardiol 50: 2215-2217. [Crossref]

55. Rowin EJ, Hausvater A, Link MS, Abt P, Gionfriddo W, et al. (2017) Clinical profile and consequences of atrial fibrillation in hypertrophic cardiomyopathy. Circulation 136: 2420-2436. [Crossref]

56. Siontis KC, Geske JB, Ong K, Nishimura RA, Ommen SR, et al. (2014) Atria fibrillation in hypertrophic cardiomyopathy: prevalence, clinical correlations, and mortality in a large high-risk population. J Am Heart Assoc 3: e001002. [Crossref]

57. Robinson K, Frenneaux MP, Stockins B, Karatasakis G, Poloniecki JD, et al. (1990) Atrial fibrillation in hypertrophie cardiomyopathy: A longitudinal study. $J$ Am Coll Cardiol 15: 1279-1285. [Crossref]

58. Ritter M, Oechslin E, Sütsch G, Attenhofer C, Schneider J, et al. (1997) Isolated noncompaction of the myocardium in adults. Mayo Clin Proc 72: 26-31. [Crossref]

59. Lyle M, Vaidya V, Kapa S, Nadipalli A, Michelena H, et al. (2018) Atrial arrhythmias in left ventricular noncompaction: Association with clinical and echocardiographic characteristics. J Am Coll Cardiol 71: A708.

60. Chu AF, Zado E, Marchlinski FE (2010) Atrial arrhythmias in patients with arrhythmogenic right ventricular cardiomyopathy/dysplasia and ventricular tachycardia. Am J Cardiol 106: 720-722.

61. Baturova MA, Haugaa KH, Jensen HK, Svensson A, Gilljam T, et al. (2020) Atrial fibrillation as a clinical characteristic of arrhythmogenic right ventricular cardiomyopathy: Experience from the Nordic ARVC Registry. Int J Cardiol 298: 39-43.

62. Wu L, Guo J, Zheng L (2016) Atrial remodeling and atrial tachyarrhythmias in arrhythmogenic right ventricular cardiomyopathy. Am J Cardiol 118: 750-753. [Crossref]

63. Mathuria N (2012) Ventricular tachycardias in the setting of cardiomyopathy: Approaches to ablation. Tex Heart Inst J 39: 529-531. [Crossref]

64. Pang BJM, Green MS (2017) Epidemiology of ventricular tachyarrhythmia. Herzschrittmacherther Elektrophysiol 28: 143-148. [Crossref]

65. Esteban MTT, García-Pinilla JM, McKenna WJ (2004) Update in arrhythmogenic right ventricular cardiomyopathy: genetic, clinical presentation, and risk stratification. Rev Esp Cardiol 57: 757-767. [Crossref]

66. Francis J, Namboodiri N (2015) Ventricular arrhythmias in arrhythmogenic right ventricular dysplasia. Indian Pacing Electrophysiol J 1: 82. [Crossref]
67. Hoffmayer K, Scheinman MM (2012) Electrocardiographic patterns of ventricular arrhythmias in arrhythmogenic right ventricular dysplasia/cardiomyopathy. Front Physiol 3: 23. [Crossref]

68. Marcus FI, McKenna WJ, Sherrill D, Basso C, Bauce B, et al. (2010) Diagnosis of arrhythmogenic right ventricular cardiomyopathy/dysplasia: proposed modification of the task force criteria. Circulation 121: 1533-1541. [Crossref]

69. Marcus FI, Zareba W, Calkins H, Towbin JA, Basso C, et al. (2009) Arrhythmogenic right ventricular cardiomyopathy/dysplasia clinical presentation and diagnostic evaluation: results from the North American Multidisciplinary Study. Heart Rhythm 6: 984-992. [Crossref]

70. Calkins H (2008) Arrhythmogenic right-ventricular dysplasia/cardiomyopathy. Curr Opin Cardiol 21: 55-63. [Crossref]

71. Sammani A, Kayvanpour E, Bosman LP, Sedaghat-Hamedani F, Proctor T, et al. (2019) P991 Predicting arrhythmic risk in dilated cardiomyopathy: a systematic review \& meta-analysis of clinical parameters. Eur Heart J 40: ehz747-0584.

72. Zecchin M, Muser D, Vitali-Serdoz L, Buiatti A, Morgera T (2019) Arrhythmias in dilated cardiomyopathy: Diagnosis and treatment. In Dilated Cardiomyopathy (pp. 149-171). Springer, Cham.

73. Spezzacatene A, Sinagra G, Merlo M, Barbati G, Graw SL, et al. (2015) Arrhythmogenic phenotype in dilated cardiomyopathy: natural history and predictors of life-threatening arrhythmias. J Am Heart Assoc 10: e002149. [Crossref]

74. Narayanan N, Francis J (2010) Ventricular arrhythmias in hypertrophic cardiomyopathycan we ever predict them? Indian Pacing Electrophysiol J 10: 112-114. [Crossref]

75. Monserrat L, Elliott PM, Gimeno JR, Sharma S, Penas-Lado M, et al. (2003) Nonsustained ventricular tachycardia in hypertrophic cardiomyopathy: an independent marker of sudden death risk in young patients. $J$ Am Coll Cardiol 42: 873-879. [Crossref]

76. Sherrid MV, Cotiga D, Hart D, Ehle F, Haas TS, et al. (2009) Relation of 12 lead electrocardiogram patterns to implanted defibrillator-terminated ventricular tachyarrhythmias in hypertrophic cardiomyopathy. Am J Cardiol 104: 1722-1726. [Crossref]

77. Ritter M, Oechslin E, Sutsch G, Attenhofer C, Schneider J, et al. (1997). Isolated noncompaction of the myocardium in adults. Mayo Clin Proc 72: 26-31. [Crossref]

78. Mattsson G, Baroudi A, Tawfiq H, Magnusson P (2017) Left ventricular noncompaction cardiomyopathy with coronary artery anomaly complicated by ventricular tachycardia. BMC Cardiovasc Disord 17: 1-5. [Crossref]

79. Rammos A, Meladinis V, Vovas G, Patsouras (2017) Restrictive Cardiomyopathies: The Importance of Noninvasive Cardiac Imaging Modalities in Diagnosis and Treatment: A Systematic Review. Radiol Res Pract: 2017. [Crossref]

80. Kennedy HL, Whitlock JA, Sprague MK, Kennedy LJ, Buckingham TA, et al. (1985) Long-term follow-up of asymptomatic healthy subjects with frequent and complex ventricular ectopy. New Eng J Med 312: 193-197. [Crossref]

81. Perez-Silva A, Luis Merino J (2011) Frequent ventricular extrasystoles: significance, prognosis and treatment. ESC Coun Cardiol Pract 9: 17.

82. Sen J, Amerena J (2019) Premature ventricular contraction-induced dilated cardiomyopathy: a case report. Eur Heart J Case Rep 3: 1-6. [Crossref]

83. Camm CF, Tichnell C, James CA, Murray B, Porterfield F, et al. (2015) Premature ventricular contraction variability in arrhythmogenic right ventricular dysplasia/ cardiomyopathy. J Cardiovasc Electrophysiol 26: 53-57. [Crossref]

Copyright: (C2020 Albakri A. This is an open-access article distributed under the terms of the Creative Commons Attribution License, which permits unrestricted use, distribution, and reproduction in any medium, provided the original author and source are credited. 